\title{
Studies on poly (hydroxy alkanoates)/(ethylcellulose) blends ${ }^{\dagger}$
}

\author{
VAISHALI SUTHAR, ARUN PRATAP* and HETA RAVAL \\ Department of Applied Chemistry, *Department of Applied Physics, Faculty of Technology and Engineering, \\ M S University of Baroda, Vadodara 390 001, India
}

\begin{abstract}
Biodegradable polymers represent one of the most significant area of research today. Among these polymers, poly ( $\beta$-hydroxy butyrate co $\beta^{\prime}$-hydroxy valerate) i.e. PHBV have received special attention because of their unique combination of properties. They are perfectly biocompatible, biodegradable polymers and can be processed by any conventional technique. In the present study an attempt has been made to develop the biodegradable blends of PHBV by blending them with ethyl cellulose (EC). Ethyl cellulose has been selected to monitor the biodegradation rate of PHBV and also for making the blends cost effective. The blends are thoroughly characterized for their compatibility, by the measurement of viscosity of blends and through FT-IR. Various applications of PHBV/EC blend in agriculture and pharmaceutical industries are being explored.
\end{abstract}

Keywords. Polymer blends; viscosity; ethyl cellulose; PHBV; compatibility.

\section{Introduction}

Polymer blends have formed a major scientific discipline of research owing to their wide range of applications. Compatibility is an important parameter (Olabishi 1975) in determining the properties of polymer blends. A number of methods has been reported measuring polymerpolymer compatibility in polymer blends. Most of them require highly sophisticated instrumentation like dynamical analysis, thermal analysis and electron microscopy and hence a great amount of technical skill (Bohmer and Florian 1970; Feldman and Rusy 1970; Paul and Newman 1978; Inamura and Jinbo 1991), whereas viscometry is a desirable alternative (Raval and Devi 1993, 1995) for studying interaction of polymers in solution. Many researchers have attempted to correlate viscosity with compatibility for ternary polymer-polymer-solvent systems. It was assumed that repulsion interaction may cause shrinkage of random coils of polymer molecule resulting in reduction in viscosities of the blends when compared with values calculated from the viscosities of the pure components, on the assumption of additivity. On the other hand, in the compatible blends, increase in viscosity due to attraction-interaction is expected. Apart from this, polymer-solvent interaction also plays an important role in changing the viscosity on blending in dilute solution blends. In the present study we have attempted to apply this approach for the study of compatibility of PHBV/EC blend systems by measuring their viscosities. The FT-IR spectroscopy further provides evidence to the

\footnotetext{
*Author for correspondence

${ }^{\dagger}$ Paper presented at the 5th IUMRS ICA98, October 1998, Bangalore
}

result obtained from the measurement of viscosities on the compatibility.

\section{Theoretical background}

Numerous expressions have been put forward for the prediction of interaction between binary polymer systems through viscosity. But, we have considered the following two approaches for the present purpose.

The viscosity behaviour of an ideally mixed polymer solution (Krigbaum and Wall 1950) can be given by

$$
\begin{aligned}
\eta_{\mathrm{sp}(\text { mix })}= & {\left[\eta_{1}\right] C_{1}+\left[\eta_{2}\right] C_{2}+b_{11} C_{1}^{2}+b_{22} C_{2}^{2} } \\
& +2\left(b_{11} b_{22}\right)^{1 / 2} C_{1} C_{2},
\end{aligned}
$$

where $\eta_{\mathrm{sp}(\mathrm{mix})}$ is the specific viscosity of the mixed polymer solution, $\eta_{1}$ and $\eta_{2}$ are intrinsic viscosities of polymer components 1 and 2 , respectively. $C_{1}$ and $C_{2}$ are concentrations of components 1 and 2 respectively in the mixed polymer solution and $b_{11}$ and $b_{22}$ are specific interaction coefficients of components in single polymer solutions.

On the other hand, a completely empirical equation was developed (Castift and Hewtt 1962).

$$
\eta_{\mathrm{sp}(\text { mix })}=\left[C_{1}\left(\eta_{\mathrm{sp}(1)}\right)_{\mathrm{c}}+C_{2}\left(\eta_{\mathrm{sp}(2)}\right)_{\mathrm{c}}\right] / C,
$$

where $\eta_{\mathrm{sp}(\mathrm{mix})}$ is the specific viscosity of the mixed polymer solution. $\left(\eta_{\mathrm{sp}(1)}\right)_{\mathrm{c}}$ and $\left(\eta_{\mathrm{sp}(2)}\right)_{\mathrm{c}}$ are specific viscosities of polymer components 1 and 2 , respectively, at concentration $C$ where $C=C_{1}+C_{2}$ and $C_{1}$ and $C_{2}$ are concentrations of components 1 and 2 , respectively in the blend system. Though this relationship does not have any theoretical justification for definition, ideal behaviour 
of many mixed polymer solutions can be quantitatively predicted by using it. In the case of polymers, the solution behaviour is described (Huggins 1942) by equation

$$
\eta_{\mathrm{sp}} / c=[\eta]+K[\eta]^{2} c
$$

If we put $K[\eta]^{2}=b$, a term which arises from polymer interaction at finite concentration, then (2) may be written as

$$
\begin{aligned}
\eta_{\mathrm{sp}(\text { mix })}= & {\left[\eta_{1}\right] C_{1}+\left[\eta_{2}\right] C_{2}+b_{11} C_{1}^{2}+b_{22} C_{2}^{2} } \\
& +\left(\left(b_{11}+b_{22}\right) / 2\right) C_{1} C_{2} .
\end{aligned}
$$

(4) is identical to (1) except for the coefficient of the $C_{1} C_{2}$ term, i.e. polymer-polymer interaction. For the convenience of computation (Krigbaum and Wall 1950), the specific interaction coefficient for the ideal behaviour of mixed polymer solutions is defined as

$$
b_{12}=\left(b_{11} b_{22}\right)^{1 / 2} \text {. }
$$

However from (4) the interaction coefficient now becomes

$$
b_{12}=\left(b_{11}+b_{22}\right) / 2 \text {. }
$$

In the case when the value of either $b_{11}$ or $b_{22}$ is negative the value of $b_{12}$ according to (5) is imaginary. Thus the Krigbaum's definition of $b_{12}$ becomes unrealistic under these conditions. Therefore, for the present purpose we have used (6) which is modified form of Castift and Hewtt's definition of ideal behaviour, throughout our work inspite of its empirical base.

Further, according to Krigbaum and Wall, information of the interaction between polymers 1 and 2 should be obtained from comparison of experimental $b_{12}$ and theoretical $b_{12}^{*}$ values. Here $b_{12}^{*}$ is referred to as the coefficient of interaction between polymers 1 and 2 as computed from (6). The compatibility of polymer mixture is predicted by a parameter

$$
\Delta b=b_{12}-b_{12}^{*} .
$$

Negative values of $\Delta b$ refer to blend solutions containing incompatible polymers while positive values of $\Delta b$ refer to compatibility of polymers due to attractive-interaction.

Table 1. Composition of various blends.

\begin{tabular}{lcc}
\hline $\begin{array}{l}\text { Code } \\
\text { no. }\end{array}$ & $\begin{array}{c}\text { Ethyl cellulose } \\
(\% \text { w/w })\end{array}$ & $\begin{array}{c}\text { PHBV } \\
(\% \text { w/w })\end{array}$ \\
\hline EC & 100 & - \\
$a_{1}$ & 90 & 10 \\
$a_{2}$ & 80 & 20 \\
$a_{3}$ & 70 & 30 \\
$a_{4}$ & 60 & 40 \\
PHBV & - & 100 \\
\hline
\end{tabular}

An alternative definition for ideal solutions may be obtained by considering the intrinsic viscosities of mixed polymer solution

$$
\left[\eta_{\mathrm{sp}(\operatorname{mix})} / c\right]_{\mathrm{c} \rightarrow 0}=\eta_{1}\left[C_{1} / C\right]_{\mathrm{c} \rightarrow 0}+\eta_{2}\left[C_{2} / C\right]_{\mathrm{c} \rightarrow 0} .
$$

The results obtained by this process are independent of the value of $b_{12}$ taken from Krigbaum and Wall or from Castift and Hewtt ((1) and (2)). Hence (2), (7), (8) can be used to predict the compatibility of PHBV/EC blends.

In the present study, attempts are made to correlate the compatibility of PHBV/EC blend systems in terms of their viscosities.

Further the effect of composition of blends on the compatibility of the system is also studied and the blends are characterized through Fourier transform infrared (FT-IR) spectroscopy.

\section{Experimental}

\subsection{Materials}

Ethyl cellulose $($ CPS epoxy content $=47 \cdot 8)(\mathrm{LR})$ was supplied by National Chemicals, Baroda, India. PHBV was gifted by G.S.F.C. Baroda, India. Dichloromethane (LR grade M.W. 84.93) and chloroform (LR grade) were supplied by Suvidhinath Laboratories, Baroda.

\subsection{Preparation of blends}

PHBV and ethyl cellulose were dissolved in dichloromethane $\left(\mathrm{CH}_{2} \mathrm{Cl}_{2}\right)$ separately. The solution of the latter was added to the former with constant stirring. Stirring was continued for $15 \mathrm{~min}$. Total concentration of the polymeric mixture in solvent was kept at 5\%. Films of polymer blends were prepared by casting the solution on the glass plate with slow drying at room temperature. Care was taken to control the uniform thickness of the film for

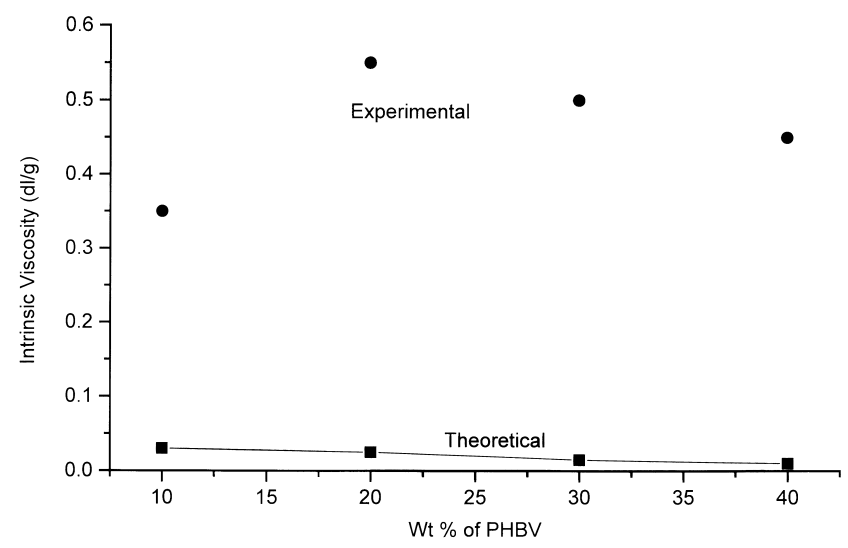

Figure 1. Intrinsic viscosities of EC/PHBV mixture versus composition. 
(a)
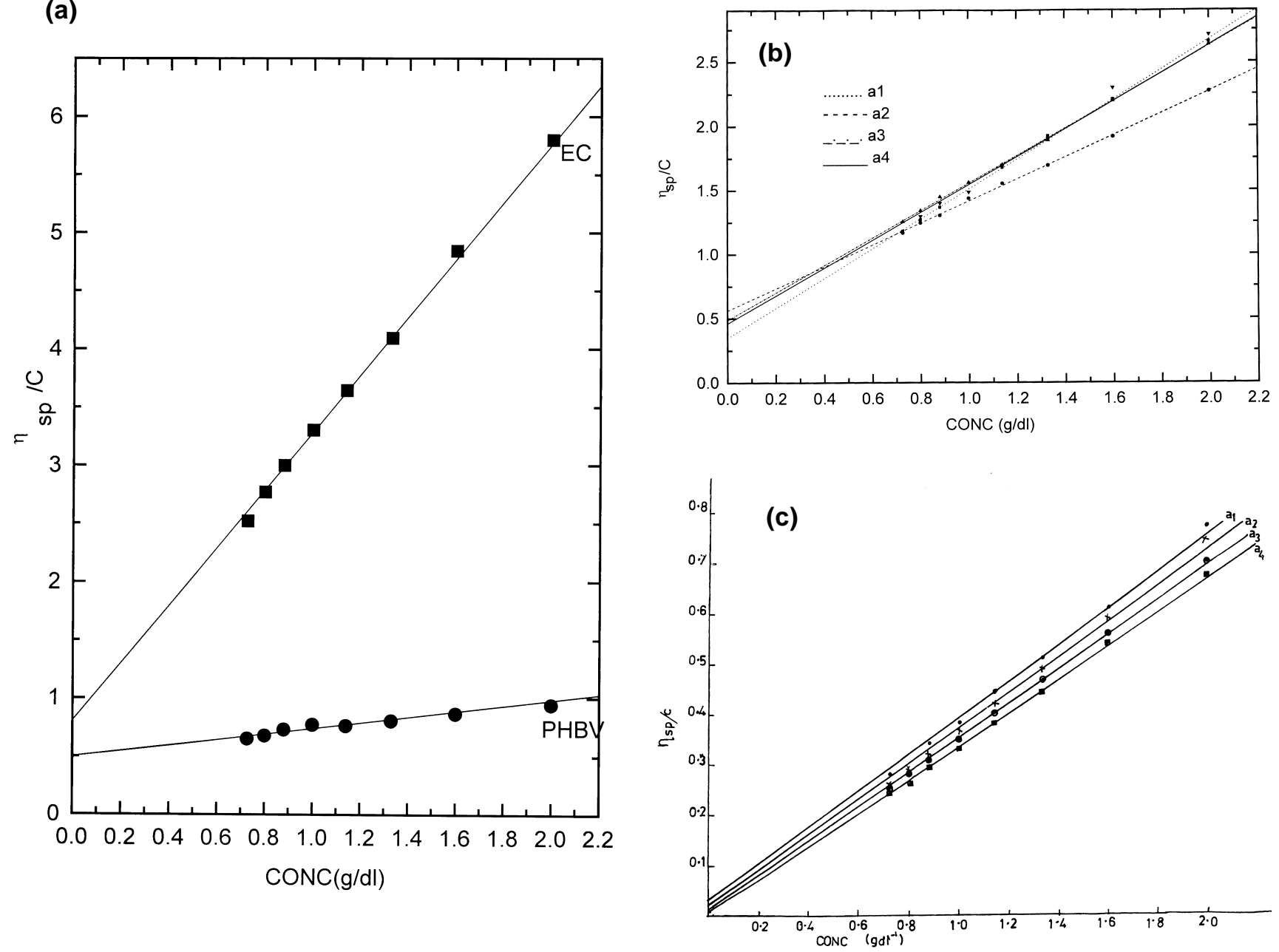

Figure 2. a. Reduced viscosity versus polymer concentration of EC and PHBV, $\mathbf{b}$. reduced viscosity versus polymer concentration of $a_{1}, a_{2}, a_{3}$ and $a_{4}$ and $\mathbf{c}$. theoretically calculated reduced viscosity versus concentration for $a_{1}, a_{2}, a_{3}$ and $a_{4}$.

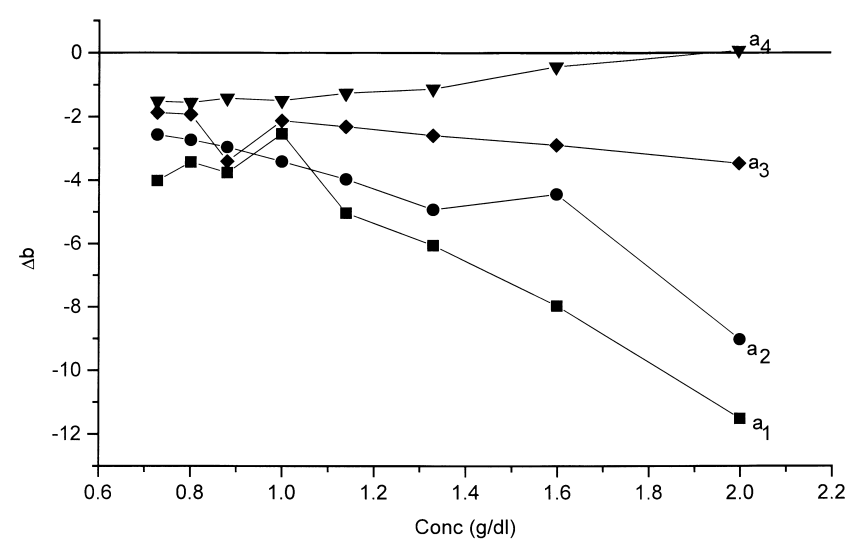

Figure 3. $\Delta b$ versus concentration for the blends. all compositions. The composition of the blends is given in table 1.

\subsection{Characterization}

The specific viscosities of the polymers and polymer blend were determined by Ubbelohde viscometer. The measurement was carried out in a closed system at $20^{\circ} \mathrm{C}$ to minimize solvent evaporation using chloroform as solvent.

IR spectroscopic information was obtained using Nicolet FT-IR and Shimadzu-14,000 Fourier transform infrared (FT-IR) instrument.

\section{Results and discussions}

The effect of the blend composition on intrinsic viscosities is illustrated in figure 1 . The straight line plot of the 
(a)

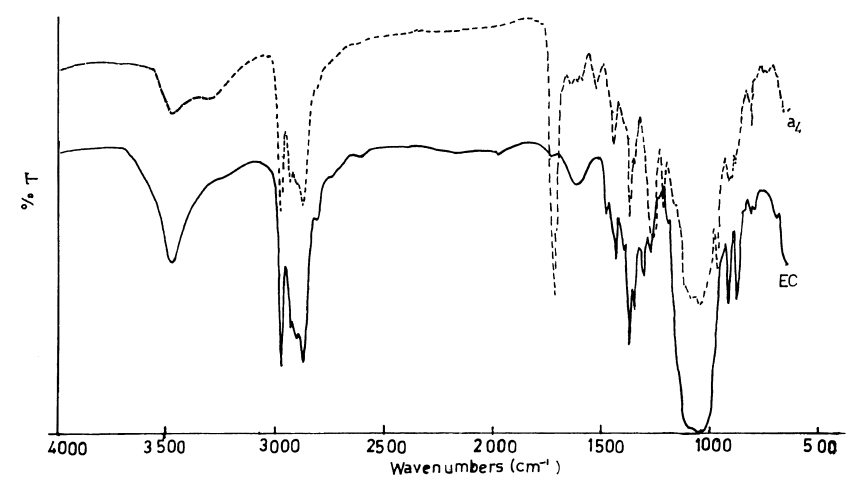

(c)

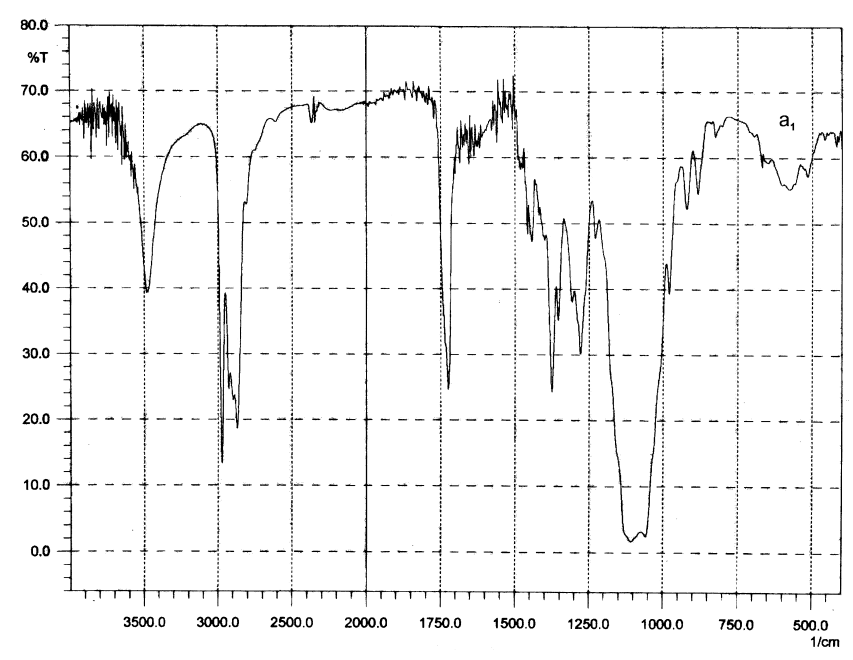

(b)

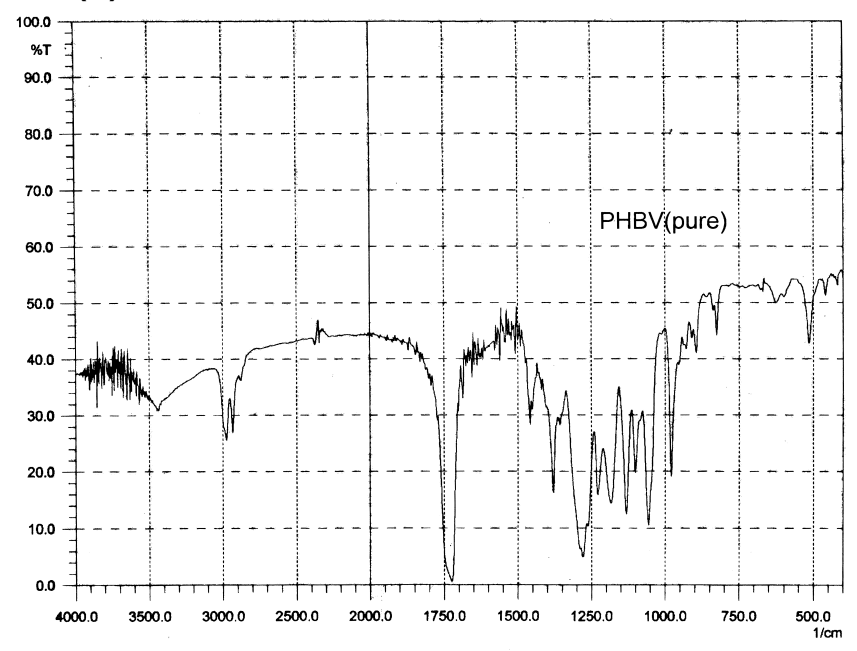

(d)

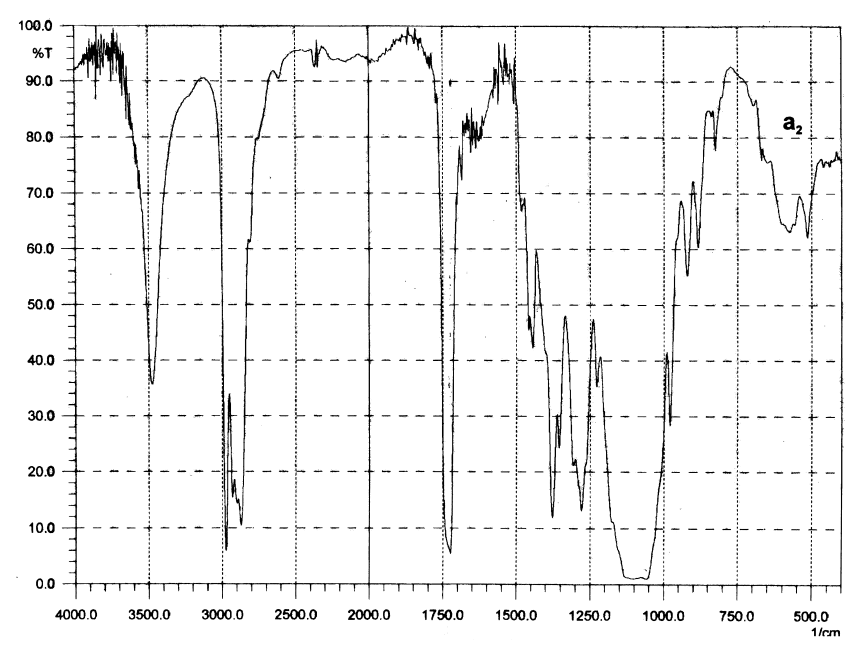

(e)

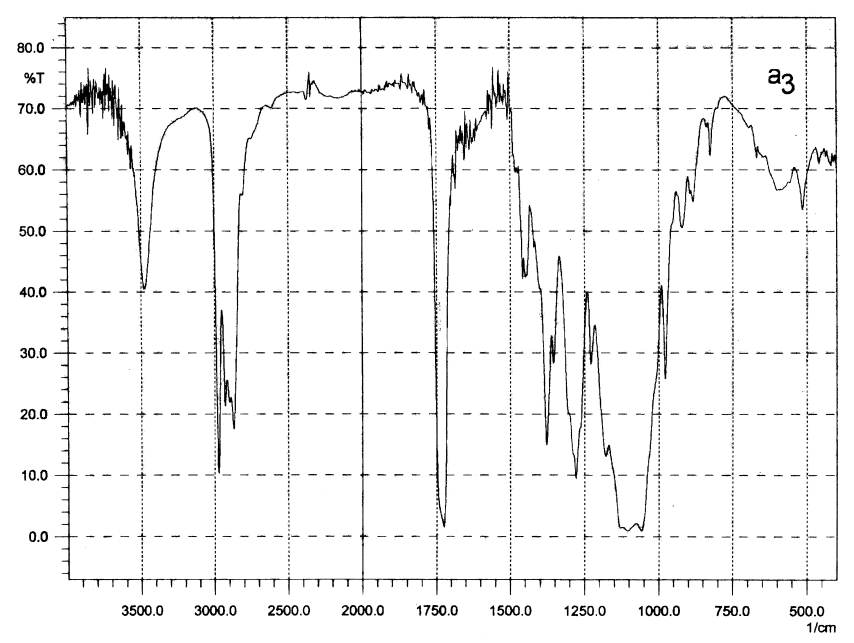

Figure 4. a. FT-IR spectra of EC and $a_{4}$, b. FT-IR spectrum of PHBV, c. FT-IR spectrum of $a_{1}$, d. FT-IR spectrum of $a_{2}$ and e. FT-IR spectrum of $a_{3}$. 
intrinsic viscosities represents ideal solution systems. The intrinsic viscosity values obtained experimentally for present system were observed to be higher indicating net attractive interaction in the blends. For the blends with 90\% (w/w) PHBV comparatively lesser deviation was observed indicating improved miscibility. However, with the increase of weight of PHBV, there is a sharp decrease in the intrinsic viscosity.

In figure $2 \mathrm{a}$ the reduced viscosities of pure PHBV and $\mathrm{EC}$ are plotted, whereas in figure $2 \mathrm{~b}$, the reduced specific viscosities for the various EC/PHBV blends and of homopolymers are presented. The same for ideally behaving compositions calculated from Castift and Hewtt (1962) (2) has been shown in figure $2 \mathrm{c}$. For all the compositions, the experimentally observed reduced viscosities shown in the single plot in figure $2 b$ and the theoretically calculated values differ in magnitude, but look very similar. The closeness of the results is due to the fact that these blends of EC and PHBV are dilute solution blends.

The plot of $\Delta b$ versus concentration of the various blends are shown in figure 3 . The negative values of $\Delta b$ for all the blends indicate repulsive interaction between the phases of the blends except $0.6: 0.4$ blends at $2 \%$ concentration. However, with increase in concentration a sharp decrease in the $\Delta b$ value is observed, indicating the existence of poor interaction between the phases of blend, here also exception is for $0.60: 0.40$ blends, in which there is sharp increase in the $\Delta b$ value as observed at higher concentration indicating existence of attractiveinteraction between the phases of the blends. At lower concentration all the blends are observed to deviate from their ideal behaviour.

The negative deviation from the ideal behaviour may be interpreted as a sign of decrease in polymer interaction. However, the overall rise in the viscosity observed experimentally is due to the dominance of polymer solvent attractive interaction over the polymer-polymer repulsion.

Thus, all the results from viscometric studies in terms of intrinsic, reduced specific viscosities and interaction between the polymer phases, indicate the immiscibility of the system except for the one composition $(0.60: 0.40$ blend).

\section{Spectroscopic studies}

Fourier transform infrared (FT-IR) spectroscopy is used to study the formulation (Ferraro and Krishnan 1990) of ethyl-cellulose-PHBV blends. The FT-IR spectra show characteristic bands for ethyl cellulose. A representative FT-IR spectrum for ethyl cellulose is shown in figure $4 \mathrm{a}$ along with the corresponding spectra of pure PHBV blended in the ratio of $0.6: 0.4$ respectively. The spectrum of ethyl cellulose shows characteristic absorption bands for $-\mathrm{C}-\mathrm{O}-\mathrm{C}-$ stretching vibration at $1052 \mathrm{~cm}^{-1}$ and $\mathrm{C}-\mathrm{H}$ stretching bands at $2880 \mathrm{~cm}^{-1}$ and $2970 \mathrm{~cm}^{-1}$. The absorption at $1369 \mathrm{~cm}^{-1}$ corresponds to $\mathrm{C}-\mathrm{H}$ bending. The FT-IR spectrum of pure PHBV (figure $4 \mathrm{~b}$ ) shows its own characteristic absorption band from $800 \mathrm{~cm}^{-1}$ to $975 \mathrm{~cm}^{-1}$ and corresponds to symmetric $-\mathrm{C}-\mathrm{O}-\mathrm{C}-$ stretching vibration. On the other hand, the antisymmetric $-\mathrm{C}-\mathrm{O}-\mathrm{C}-$ stretching leads to bands between $1060 \mathrm{~cm}^{-1}$ and $1150 \mathrm{~cm}^{-1}$. The absorption band at $1725 \mathrm{~cm}^{-1}$ is due to the $\mathrm{C}=\mathrm{O}$ stretching in PHBV. The FT-IR spectrum of blend of EC and PHBV (0.6: $0.4 \mathrm{wt} / \mathrm{wt})$ depicted as $a_{4}$ shown in figure $4 \mathrm{a}$ exhibits almost all the features of both ethyl cellulose and PHBV as they individually show. The absorption peaks of ethyl cellulose at $1052 \mathrm{~cm}^{-1}$ due to C-O-C stretching and at $2880 \mathrm{~cm}^{-1}$ and $2970 \mathrm{~cm}^{-1}$ corresponding to $\mathrm{C}-\mathrm{H}$ stretching may be easily observed apart from band at $3500 \mathrm{~cm}^{-1}$ owing to $\mathrm{O}-\mathrm{H}$ stretching. The characteristic absorption bands from $1060 \mathrm{~cm}^{-1}$ to $1150 \mathrm{~cm}^{-1}$ are clearly visible in the blend. The typical absorption band at $1725 \mathrm{~cm}^{-1}$ caused by the $\mathrm{C}=\mathrm{O}$ stretching of PHBV is also present in the blend. All the other blends i.e. $a_{1}, a_{2}$ and $a_{3}$ more or less show the similar observation of absorption bands in the figures $4 \mathrm{c}, 4 \mathrm{~d}$ and $4 \mathrm{e}$ respectively except for the variation in the intensities of the bands due to difference in their composition. It may also be observed that there is practically no shift in the characteristic absorption bands EC and PHBV after blending showing incompatibility.

\section{Conclusion}

In general, PHBV shows incompatibility with ethyl cellulose both from viscosity measurements and also from spectroscopic studies. Only for a particular composition EC : PHBV (60:40), viscosity studies show attractive interaction indicating improved miscibility.

\section{References}

Bohmer B and Florian S 1970 Eur. Polym. J. 6471

Castift E H and Hewtt W A 1962 J. Appl. Polym. Sci. 6 530, 962

Feldman D and Rusy M 1970 Eur. Polym. J. 6627

Ferraro J R and Krishnan K (eds) 1990 Practical Fourier transform infrared spectroscopy (New York: Academic Press)

Huggins M L 1942 J. Am. Chem. Soc. 642716

Inamura I and Jinbo Y 1991 Polym. J. 231143

Krigbaum W R and Wall F T 1950 J. Polym. Sci. 5505

Olabishi O 1975 Macromolecules 8317

Paul D R and Newman S 1978 Polymer blends (New York: Academic Press)

Raval Heta and Devi Surekha 1993 Polym. J. 251215

Raval Heta and Devi Surekha 1995 Die Ang. Makromol. Chem. 22727 